\title{
Brief Intervention for Tobacco when Diagnosed with Oral Cancer (BITDOC): Study protocol of a randomized clinical trial studying efficacy of brief tobacco cessation intervention, Chhattisgarh, India
}

\author{
Lokesh K. Singh ${ }^{1}$, Ripu Daman Arora ${ }^{2}$, Sai Krishna Tikka ${ }^{1}$, Avinash Shukla ${ }^{1}$, Sharda Singh ${ }^{1}$, Supriya Mahant ${ }^{1}$, Sachin \\ Verma ${ }^{1}$
}

\begin{abstract}
INTRODUCTION Tobacco use is a major causative factor for cancer. Cessation programs along with diagnosis of cancer as a motivating factor may improve quitting rates in patients. This is a protocol of a study that aims to assess the efficacy of brief tobacco cessation intervention (compared to treatment as usual, TAU) on pattern and attitudes towards tobacco chewing in newly diagnosed head and neck cancer patients and their relatives.

METHODS The proposed study will be conducted in two phases. Phase 1 will include 105 dyads of patients and relatives and shall assess patterns (amount, frequency, duration of use and dependence etc.) and knowledge and attitudes (quitting, continued use, health-behavioural modifications, long-term effects on treatment etc.) towards tobacco chewing in newly diagnosed head and neck cancer patients using smokeless tobacco and their relatives. Sample will be recruited from outpatients attending the ear, nose, throat and head and neck surgery department of a tertiary health care institute. Phase 2 will be a randomized trial that will compare the efficacy of the 'Brief Intervention for Tobacco when Diagnosed with Oral Cancer' (BITDOC) and TAU, and will include 27 dyads in each of the two groups. Intervention will be delivered in three sessions, based on the principles of motivational interviewing and the 3As model.

CONCLUSIONS This study will help in the evaluation of the attitude towards smokeless tobacco (SLT) in a population that has faced the adverse consequences from its use and changes brought by a diagnosis of HNC. It will also help in developing a cost-effective model for promotion of smoking cessation.
\end{abstract}

AFFILIATION

1 Department of Psychiatry, AllMS, Raipur, India

2 Department of ENT \& Head and Neck Surgery, AIIMS, Raipur, India

CORRESPONDENCE TO

Avinash Shukla. Department of Psychiatry, AlIMS, Raipur

Chhattisgarh, India. E-mail: mailto:dravinashcip@gmail.com

KEYWORDS

head and neck cancer, smokeless tobacco, brief intervention, tobacco cessation interventions

Received: 29 September 2019 Revised: 29 November 2019 Accepted: 3 December 2019

\section{INTRODUCTION}

Tobacco use is a major causative factor for cancer. Continued use of tobacco even after cancer diagnosis has been found to be associated with various poor prognostic factors, i.e. second primary cancer, all-cause and cancer-specific mortality, cancer recurrence, poor treatment response, and treatment-related toxicity ${ }^{1-4}$. The psychological impact of continued tobacco use adds to already prevailing depression in patients and overall to the family's impaired functioning caused by the diagnosis of cancer ${ }^{5}$.

Time of cancer diagnosis has been termed as a 'teachable moment' for tobacco cessation, as diagnosis of cancer personalizes harms of tobacco use and directs priorities to restoration and maintenance of good health for patients and 
their loved ones using tobacco. On the other hand, severe nicotine dependence, urgency of cessation, fatalistic attitudes about cessation benefits, cancerrelated psychological distress, treatment factors, and the presence of tobacco users in the social network have been identified as barriers to this 'moment' ${ }^{6}$. Apart from diagnosis of cancer being a motivational factor to quit tobacco use, a conjunction with tobacco cessation programs that target these factors may improve quitting rates in patients. For these reasons smoking cessation interventions have been recommended as part of standard oncologic treatment ${ }^{7}$.

A few systematic reviews and meta-analyses assessing the efficacy of tobacco cessation intervention in cancer patients, in genera ${ }^{8-10}$, as well as in head and neck cancer (HNC) patients, specifically ${ }^{11-13}$, have been conducted. The meta-analysis by Klemp et al. ${ }^{11}$ showed smoking cessation was achieved considerably more often in HNC patients who received cessation counselling compared with those receiving usual care. The majority of the literature on clinical impact of continued tobacco use after diagnosis has been on tobacco smoking. Among the randomized controlled trials assessing tobacco cessation in cancer patients, in general $^{14-21}$, as well as in HNC patients specifically ${ }^{22-24}$, none has focused on patients using smokeless tobacco (SLT).

Intriguingly, significant changes in the attitudes and behaviours of patients' relatives toward cancer prevention and screening occur after the patients are diagnosed with cancer ${ }^{25}$. However, Sarna et al. ${ }^{26}$ reported that even though higher quit rates were seen in cancer patients, quit rates were lower among family members who smoked. Expanding the benefits, therefore, to psychological interventions targeting tobacco use in relatives along with those diagnosed with cancer may be beneficial. Moreover, evidence suggests that continued smoking undermines patients' individual efforts to quit and hence there is a need to consider the patientfamily member dyad in the context of tobacco cessation $^{27-28}$. Studies using dyadic involvement with targeted education of the family member regarding illness state and impact of smoking on health have found better patient engagement with quitline services $^{29}$, overall better smoking cessation rates ${ }^{30}$ and increased supportive behaviour of the family members towards the patient ${ }^{31}$.

In the state of Chhattisgarh, India, the prevalence of tobacco-use disorders $(29.9 \%)$ is above the national prevalence $(20.9 \%)^{32}$, with $47.7 \%$ of men, $24.5 \%$ of women, and $36.0 \%$ of all adults, currently using $\mathrm{SLT}^{33}$. This is 5 times greater than rates of tobacco smoking. Notably, National Cancer Registry Programme (NCRP, 2012-14) reports $45.7 \%$ and $16.5 \%$ ( $43.4 \%$ and $13.2 \%$, in the state of Chhattisgarh) of cancers to be tobacco related cancers, in males and females, respectively ${ }^{34}$. Of these $48.55 \%$ in males and $41.82 \%$ in females have head and neck cancers (HNC: lip, tongue, mouth, oropharynx, hypopharynx, pharynx, and larynx), which have been associated with use of $\mathrm{SLT}^{35}$. Primary objective of the proposed study is to assess the efficacy of a brief tobacco cessation intervention (compared to treatment as usual) on cessation rates of tobacco chewing in newly diagnosed HNC patients and their relatives. Changes in other patterns (such as amount, frequency, dependence etc.) and knowledge and attitudes (towards association of SLT and cancer, quitting, continued use, towards health warnings, health-behavioral modifications, long-term consequences on treatment etc.) towards tobacco chewing will be secondary outcome variables. An ancillary objective of the study is to find out the effect of diagnosis of HNC on cessation rates, patterns and attitudes towards tobacco chewing in patients and their relatives, and to determine predictors of post intervention, change in attitude and pattern towards SLT use. Here, we present the rationale for the study, the study design and its protocol.

\section{METHODS}

This current study protocol follows the SPIRIT 2013 Statement, which provides recommendations for a minimum set of scientific, ethical and administrative elements that should be addressed in a clinical-trial protocol.

\section{Study design}

The study will be conducted in two phases. Phase 1 is a cross-sectional, descriptive, questionnaire-based study of patients and their relatives attending the outpatient/inpatient services. From the total number of participants recruited, a group of participants will 
be enrolled for the Brief Intervention for Tobacco the study design is presented in Figure 1. The study when Diagnosed with Oral Cancer (BITDOC), has the approval of the institutional ethics committee i.e. the second phase. Phase 2 is a longitudinal, and has been registered at ctri.nic.in (identifier: randomized control trial study. A flow diagram of CTRI/2019/02/017738).

\section{Figure 1. Flow diagram of study design}

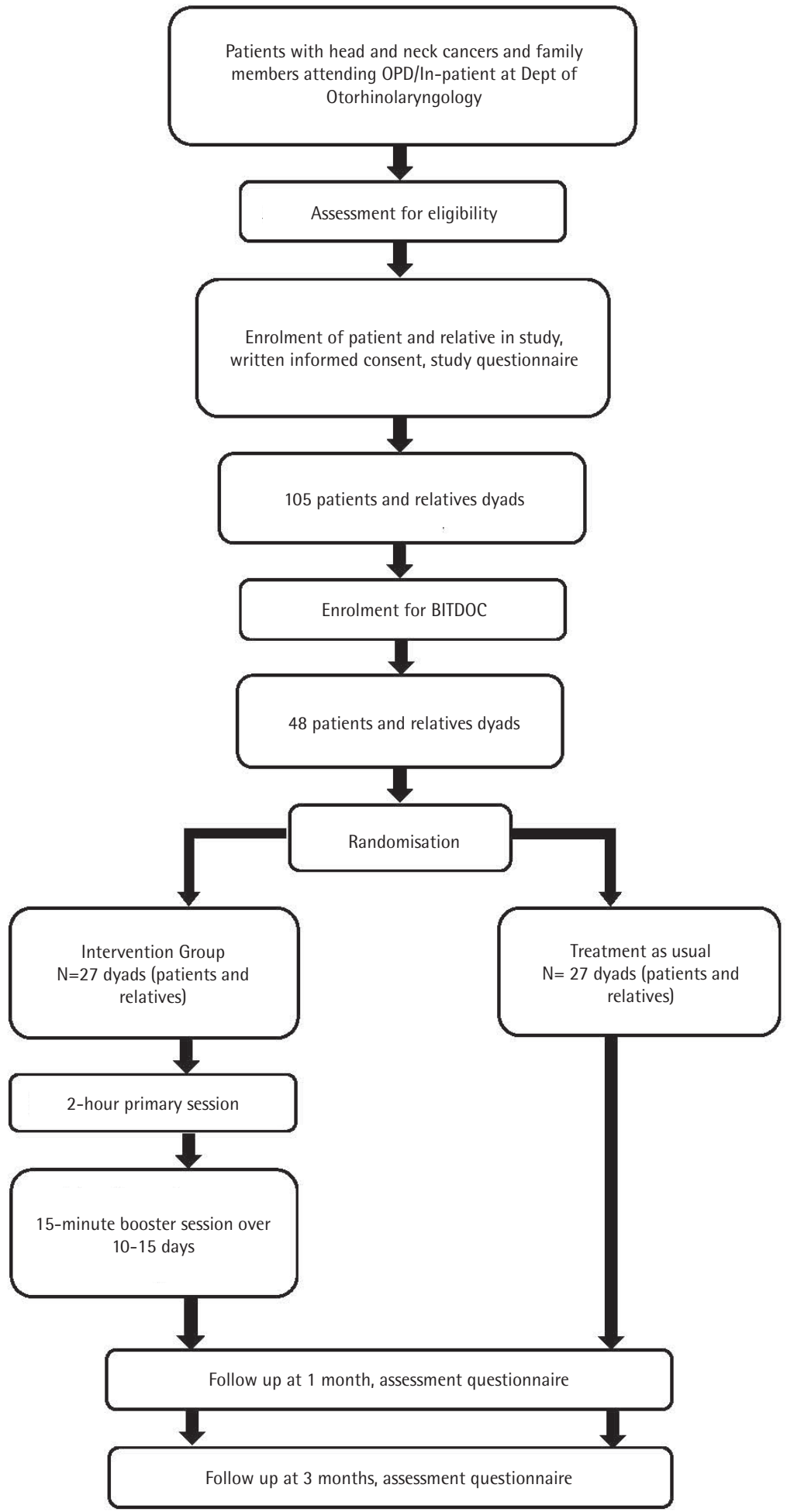




\section{Study settings}

The study began in January 2019 at the All India Institute of Medical Sciences (AIIMS), Raipur, Chhattisgarh, India, which is a tertiary care centre offering speciality services to patients belonging to the states of Chhattisgarh, Odisha, adjoining regions of Maharashtra, Madhya Pradesh, and Jharkhand. The current study is being conducted by the Department of Psychiatry in collaboration with the department of Otorhinolaryngology. Sample will be recruited from outpatients attending the department of Otorhinolaryngology. The study is designed to be completed by one year from the start of the project.

\section{Participants}

The study will include patients recently diagnosed with head and neck cancers; with a history of current use (continued use for at least 6 months before the diagnosis of cancer) of smokeless tobacco, who are aged $>18$ years, and belong to either gender. Family members to be included in the study should have lived with the patient for at least one year. Family members who are currently pregnant or diagnosed with severe mental disorders like psychotic disorders, obsessive compulsive disorder, dementia, or with a past diagnosis and treatment for any cancer or precancerous lesions/conditions will be excluded from the study. Family members who are not SLT users will also be able participate in the study. The rationale being to assess attitudes towards SLT use and enhance them during the intervention. Support person (non-users) intervention has been found to be effective in promoting quitting in users ${ }^{29}$.

\section{Sample size}

Based on the findings of Koca et al. ${ }^{25}$, i.e. $37.4 \%$ proportion of the relatives having a change in their attitudes and behaviors toward prevention and screening of cancer, we calculated an a priori sample size. With a $25 \%$ relative margin of error, the sample size, $n$, required to estimate the given proportion was found to be about 105, calculated from the expression:

$$
\begin{aligned}
& n=\left(Z^{2} \times p(1-p)\right) / e^{2} \\
& =\left(1.96^{2} \times 0.374(1-0.374)\right) /\left(0.0935^{2}\right) \\
& =102.88(\sim 103)
\end{aligned}
$$

Where $\mathrm{Z}$ is the level of confidence at $95 \%$, e is the tolerated margin of error and $p$ is the estimated proportion of the population. Tentatively, in Phase
1, we intend to have an analysable sample of 105 patients and 105 relatives. For Phase 2, sample size calculation was done keeping $90 \%$ power and error probability of 5\% (2-tailed), and effect size (d) of 0.345 (meta-analysis by Klemp et al. ${ }^{11}$ ). With correlation between the repeated measures of zero, the estimated total sample size required is 48 . With an expected $10 \%$ dropout in the sample, total calculated sample size in each of the two groups is 53 ; hence, a minimum of 27 dyads of patients and relatives will be recruited.

\section{Recruitment of participants}

The participants in the study will be recruited using a purposive sampling method. All the participants recruited as part of Phase 1 will be approached to be part of Phase 2. For Phase 2, the participants will be randomized into two subgroups: A - BITDOC; B 'Treatment as usual (TAU)'. Blocked randomization with fixed block sizes of four will be used. A concealed allocation schedule using sealed opaque envelops will be undertaken for allocating patients in the two groups.

\section{Intervention}

Intervention will be delivered by trained mental health professionals having adequate experience in psychotherapy. The BITDOC shall be based on the principles of motivational interviewing ${ }^{36}$ and the 3 As model ${ }^{37}$. The 3As (Ask cancer patients about their tobacco use; Advise patients about the benefits of quitting; Act to refer the patient to a smoking cessation program) model has been found to be suited for delivering tobacco treatment in primary care practice $^{38}$. Further, a recent study has recommended this model for tobacco cessation in cancer patients ${ }^{7}$. The intervention shall consist of a 2-hour primary session and a 15-minute booster (refresher) session spaced over 10-15 days. The primary session will be conducted in an individual (dyad) therapy mode and the booster session will be in an individual format (telephonic mode may be used when in-person sessions not feasible). The contents of intervention will be as described in Table 1 .

\section{Outcome variables and their assessment}

A study-specific questionnaire evaluating different variables as mentioned below has been developed for use in this study. 


\section{Table 1. Steps and components of the intervention}

Steps
One (Ask) Introduction to the session with positive feedback on patient's willingness to engage in the intervention,
expression of interest and concern transporting a positive and empathic therapeutic mindset and explanation of the
intervention's aim and content.
- A semi-structured interview assessing circumstances of SLT use behaviors.
Two (Advice)
- Demonstration of impact of SLT on oral health, consequences of continued SLT use post-cancer diagnosis etc., using
slide shows (containing scientific data and media reports).
- Exploration phase incorporating discussion (primary focus being sharing experiences) on attitudes and patterns of
SLT use (especially in the context of HNC) with optional use of tools (decisional balance sheets) and establishment of
future scenarios with changed/unchanged SLT use.
- Summary in which the facilitator structures and sums up what has been discussed, highlights personal responsibility
for change and asks the patient for his/her conclusion from what has been discussed so far.
- Closing session shall begin with identification of SLT goals and potential barriers and development of strategies
for goal attainment. The session is finished with a written agreement on SLT goals, introduction of various
pharmacological options for tobacco cessation including nicotine replacement therapy (NRT), introduction of various
long-term psychological interventions including cognitive behavioral therapy and family therapy, referral, if required,
to these clinics and, promotion of patient's self-efficacy.

SLT: smokeless tobacco.

\section{Independent variables}

- Pre-diagnosis and post-diagnosis pattern of SLT use: present/absent, types (betel quid with tobacco, khaini, gutka, oral tobacco application, pan masala with tobacco), amount, total duration, dependence (present/absent), duration in dependence pattern, severity of dependence, attempts at abstinence, period of abstinence, average daily cost, psychological co-morbidity (depression, anxiety, impaired quality of life, psychological distress) etc.

- Pre-diagnosis and post-diagnosis 'knowledge and attitude' towards SLT use based on whether: it leads to cancer; they saw pictorial health warnings on packets; pictorial health warnings on packets appropriate; pictorial health warnings on packets have significant impact; the size of the pictorial health warnings on packets be enlarged; they saw/heard/read health warnings aired/printed in media; health warnings aired/printed in media appropriate; health warnings aired/printed in media have significant impact; the frequency of health warnings aired/printed in media be increased; attempted to quit SLT use; attempted to reduce SLT use.

- 'Knowledge and Attitude' towards long-term consequences with SLT, based on whether: it can aggravate cancer or cause recurrence; it can cause cancer elsewhere; it interferes with treatment; continued use can lead to earlier death; it can aggravate psychological co-morbidity (depression, anxiety, impaired quality of life, psychological distress); there is urgent need to quit tobacco; there is a further worth in quitting tobacco; benefits of quitting tobacco outweigh difficulties of quitting.

- Attitude towards 'health behavioural' modifications: pattern of comorbid tobacco smoking and any other substances (similar to pattern of SLT); attitude change in eating habits, physical exercise, other lifestyle factors (stress, leisure, holidaying, shopping, spending time with family and friends); screening for cancer; use of alternative/ complementary therapies and/or vitamins for protection against cancer etc.

- Disease-related factors (only for patient): cancer site; stage (including precancerous stages); comorbidities; and treatment (surgery and radiation/chemotherapy, duration, side-effects, prophylaxis).

Outcome variables shall be applied before (1-3 days) the commencement of intervention (assessed as part of 'impact of cancer diagnosis' phase), at one and three months after the intervention. Principally, the follow-up assessments shall be conducted in person. However, when not feasible, assessments over telephone conversations shall be carried out. Outcome 
assessments shall be blinded to the intervention group allocation and conducted by the person collecting data in the 'impact of cancer diagnosis' phase.

One person (research assistant) shall rate the outcomes and he/she shall be blind to randomization/allocation schedules. Person providing the intervention will not aid any outcome assessments.

\section{Assessment tools/Instruments}

A study-specific questionnaire will be used for assessment and evaluation of patients and their relatives in the study. The questionnaire will be based on variables assessed across various relevant studies and other specific domains identified in the literature review.

\section{Study specific questionnaire}

a. SLT (type, duration, amount): Global Adult Tobacco Survey 2 (GATS 2) $)^{33}$;

b. Severity of tobacco dependence: A modified version of Fagerström developed to assess severity of dependence of smokeless tobacco will be used ${ }^{39-40}$;

c. Attitude towards health warnings: Adopted and extended based on Ahsan et al. ${ }^{41}$;

d. Change in attitudes towards health-behavior modifications (substance, physical exercise, food habits, lifestyle etc.) towards cancer prevention and screening (Koca et al. ${ }^{25}$ );

e. Knowledge and attitude towards long-term consequences with SLT related to cancer progression and management (McBride and Ostroff6, Choi et al. ${ }^{2}$ ).

\section{Tumour stage $(0-I V)$}

Tumor stage will be assessed using the American Joint Committee on Cancer (AJCC) staging classification system $^{42}$.

\section{Comorbidities}

Comorbidities will be assessed by the Adult Comorbidity Evaluation-27 (ACE-27) and classified as none, mild, moderate, or severe ${ }^{43}$.

Measurement of oral health related quality of life (OHRQoL)

A Hindi translated version ${ }^{44}$ of the Oral Health Impact Profile (OHIP-14) ${ }^{4}$ will be used in the study.

\section{Statistical analysis}

Frequencies and descriptive statistics shall be calculated and compared using independent samples t-test or chi-squared test. Pearson/Kendall tau correlation coefficients and corresponding partial correlation coefficients shall be calculated for correlation assessment. A step-by-step discriminant function analysis using Wilk's lambda method shall be used for prediction analysis. A $3 \times 2$ repeated measures ANOVA will be used to analyse the pre-post effects of the interventions on the outcome variables. 'Both patient-relative use SLT' and 'only patient uses SLT' covariation within the dyads will be corrected in the analysis.

\section{DISCUSSION}

In middle-income countries, like India, it is prudent that studies are conducted to assess the attitude of population towards various tobacco products, especially SLT which has a higher prevalence compared to other forms. It is also important to test various models that are cost effective, less intensive and addressing various barriers for promotion of tobacco cessation. Hence, a study like this is of utmost importance. This will help evaluation of not just the knowledge and attitude towards SLT in a population that has faced adverse consequences from the use to SLT but also for assessment of changes brought about by diagnosis of HNC. This study will further help in creating a model that can be used for promotion of smoking cessation in such populations.

\section{Limitations}

The sample size estimation for the randomized trial phase of the study is based on effect size of interventions using CBT and counselling, with most of the studies including NRT also, for smoking cessation and hence it is an extrapolation. Therefore, effect sizes used for sample size estimation in the study may be overestimated.

More importantly, use of the 3As model might limit the individual efficacy compared to other models such as CBT, 5As etc., especially with regard to high rates of tobacco use and potential lack of knowledge and negative attitudes towards tobacco cessation. However, keeping the resource limited (in terms of a very large mental health treatment gap) ${ }^{32}$ settings in India, we believe the brief intervention 
model using 3As should fare well in terms of feasibility for use on community scale.

\section{CONCLUSION}

This is a first study in India for the evaluation of a brief intervention for tobacco cessation in cancer patients. There is a need for conducting such studies at a larger scale for evaluation of effectiveness of brief intervention in populations at risk. If effective, this model may bring about expected changes towards tobacco cessation without needing intensive therapy, especially in places where there are fewer trained clinicians and specialists.

\section{REFERENCES}

1. Burris JL, Studts JL, DeRosa AP, Ostroff JS. Systematic Review of Tobacco Use after Lung or Head/Neck Cancer Diagnosis: Results and Recommendations for Future Research. Cancer Epidemiol Biomarkers Prev. 2015;24(10):1450-1461. doi:10.1158/1055-9965.EPI-15-0257

2. Choi SH, Terrell JE, Bradford CR, Ghanem T, Spector ME, Wolf GT et al. Does Quitting Smoking Make a Difference Among Newly Diagnosed Head and Neck Cancer Patients? Nicotine Tob Res. 2016;18(12):22162224. doi:10.1093/ntr/ntw189

3. Garces YI, Yang P, Parkinson J, Zhao X, Wampfler JA, Ebbert JO et al. The relationship between cigarette smoking and quality of life after lung cancer diagnosis. Chest. 2004;126(6):1733-1741. doi:10.1378/chest.126.6.1733

4. Petros WP, Younis IR, Ford JN, Weed SA. Effects of tobacco smoking and nicotine on cancer treatment. Pharmacotherapy. 2012;32(10):920-931. doi:10.1002/j.1875-9114.2012.01117

5. Edwards B, Clarke V. The psychological impact of a cancer diagnosis on families: the influence of family functioning and patients' illness characteristics on depression and anxiety. Psychooncology. 2004;13(8):562-576. doi:10.1002/pon.773

6. McBride CM, Ostroff JS. Teachable moments for promoting smoking cessation: the context of cancer care and survivorship. Cancer Control. 2003;10(4):325-333. doi:10.1177/107327480301000407

7. Davidson SM, Boldt RG, Louie AV. How can we better help cancer patients quit smoking? The London Regional Cancer Program experience with smoking cessation. Curr Oncol. 2018;25(3):226-230. doi:10.3747/co.25.3921

8. Nayan S, Gupta M, Sommer DD. Evaluating smoking cessation interventions and cessation rates in cancer patients: a systematic review and meta-analysis. ISRN Oncol. 2011;2011:849023. doi:10.5402/2011/849023

9. Nayan S, Gupta MK, Strychowsky JE, Sommer DD. Smoking cessation interventions and cessation rates in the oncology population: an updated systematic review and meta-analysis. Otolaryngol Head Neck Surg. 2013;149(2):200-211. doi:10.1177/0194599813490886

10. Lucchiari C, Masiero M, Botturi A, Pravettoni G. Helping patients to reduce tobacco consumption in oncology: a narrative review. Springerplus. 2016;5(1):1136. doi:10.1186/s40064-016-2798-9

11. Klemp I, Steffenssen M, Bakholdt V, Thygesen T, Sørensen JA. Counseling is Effective for Smoking Cessation in Head and Neck Cancer Patients-A Systematic Review and Meta-Analysis. J Oral Maxillofac Surg. 2016;74(8):16871694. doi:10.1016/j.joms.2016.02.003

12. McGarter K, Martínez Ú, Britton B, Baker A, Bonevski B, Carter G, Beck A, Wratten C, Guillaumier A, Halpin SA, Wolfenden L. Smoking cessation care among patients with head and neck cancer: a systematic review. BMJ Open. 2016;6(9):e012296. doi:10.1136/bmjopen-2016-012296

13. Shingler E, Robles LA, Perry R, Penfold C, Ness AR, Thomas S, et al. Systematic review evaluating randomized controlled trials of smoking and alcohol cessation interventions in people with head and neck cancer and oral dysplasia. Head Neck. 2018;40(8):1845-1853. doi:10.1002/hed.25138

14. Stanislaw AE, Wewers ME. A smoking cessation intervention with hospitalized surgical cancer patients: a pilot study. Cancer Nurs. 1994;17(2):81-86. doi:10.1097/00002820-199404000-00001

15. Griebel B, Wewers ME, Baker CA. The effectiveness of a nurse-managed minimal smoking-cessation intervention among hospitalized patients with cancer. Oncol Nurs Forum. 1998;25(5):897-902. PMID:9644706.

16. Schnoll RA, Zhang B, Rue M, Krook JE, Spears WT, Marcus AC et al. Brief physician-initiated quit-smoking strategies for clinical oncology settings: a trial coordinated by the Eastern Cooperative Oncology Group. J Clin Oncol. 2003;21(2):355-365. doi:10.1200/JC0.2003.04.122

17. Wakefield M, Olver I, Whitford H, Rosenfeld E. Motivational interviewing as a smoking cessation intervention for patients with cancer: randomized controlled trial. Nurs Res. 2004;53(6):396-405. doi:10.1097/00006199-200411000-00008

18. Schnoll RA, Rothman RL, Wielt DB, Lerman C, Pedri H, Wang $\mathrm{H}$ et al. A randomized pilot study of cognitivebehavioral therapy versus basic health education for smoking cessation among cancer patients. Ann Behav Med. 2005;30(1):1-11. doi:10.1207/s15324796abm3001_1

19. Schnoll RA, Martinez E, Tatum KL, Weber DM, Kuzla $\mathrm{N}$, Glass M, et al. A bupropion smoking cessation clinical trial for cancer patients. Cancer Causes Control. 2010;21(6):811-820. doi:10.1007/s10552-010-9507-8

20. Thomsen T, Tønnesen H, Okholm M, Kroman N, Maibom A, Sauerberg ML, et al. Brief smoking cessation intervention in relation to breast cancer surgery: a randomized controlled trial. Nicotine Tob Res. 2010;12(11):1118-1124. doi:10.1093/ntr/ntq158

21. Ostroff JS, Burkhalter JE, Cinciripini PM, Li Y, Shiyko 
MP, Lam CY, et al. Randomized trial of a presurgical scheduled reduced smoking intervention for patients newly diagnosed with cancer. Health Psychol. 2014;33(7):737-747. doi:10.1037/a0033186

22. Gritz ER, Carr CR, Rapkin D, Abemayor E, Chang LJ, Wong WK, et al. Predictors of long-term smoking cessation in head and neck cancer patients. Cancer Epidemiol Biomarkers Prev. 1993;2(3):261-270. PMID:8318879.

23. Duffy SA, Ronis DL, Valenstein M, Lambert MT, Fowler KE, Gregory L et al. A tailored smoking, alcohol, and depression intervention for head and neck cancer patients. Cancer Epidemiol Biomarkers Prev. 2006;15(11):22032208. doi:10.1158/1055-9965.EPI-05-0880

24. Ghosh A, Philiponis G, Bewley A, Ransom ER, Mirza $\mathrm{N}$. You can't pay me to quit: the failure of financial incentives for smoking cessation in head and neck cancer patients. J Laryngol Otol. 2016;130(3):278-283. doi:10.1017/S0022215116000037

25. Koca D, Oztop I, Yilmaz U. Evaluation of changes in the attitudes and behaviors of relatives of lung cancer patients toward cancer prevention and screening. Indian J Cancer. 2013;50(3):233-238. doi:10.4103/0019-509X.118740

26. Sarna L, Cooley M, Brown J, Williams R, Chernecky C, Padilla G, et al. Quality of Life and Health Status of Dyads of Women With Lung Cancer and Family Members. Oncol Nurs Forum. 2006;33:1109-1116. doi:10.1188/06.ONF.1109-1116

27. Schnoll R, Malstrom M, James C, Rothman R, Miller S, Ridge J, et al. Correlates of tobacco use among smokers and recent quitters diagnosed with cancer. Patient Educ Couns. 2002;46(2):137-145. doi:10.1016/S0738-3991(01)00157-4

28. Weaver KE, Rowland JH, Augustson E, Atienza AA. Smoking Concordance in Lung and Colorectal Cancer Patient-Caregiver Dyads and Quality of Life. Cancer Epidemiol Biomarkers Prev. 2011;20(2):239-248. doi:10.1158/1055-9965.EPI-10-0666

29. Patten CA, Boyle R, Tinkelman D, Brockman TA, Lukowski A, Decker PA, et al. Linking smokers to a quitline: randomized controlled effectiveness trial of a support person intervention that targets nonsmokers. Health Educ Res. 2017;32(4):318-331. doi:10.1093/her/cyx050

30. McCarthy MJ, Craddock WS, Acquavita SP, Black K. A mixed-methods study of smoking attitudes and behaviors among dual-smoker stroke survivor-caregiver dyads. J Health Psychol. 2016;23(13):1659-1667. doi:10.1177/1359105316667797

31. Chan S, Cheung YTD, Fong D, Emmons K, Leung A, Leung $D$, et al. Family-Based Smoking Cessation Intervention for Smoking Fathers and Nonsmoking Mothers with a Child: A Randomized Controlled Trial. J Pediatr. 2016;182:260266.e4. doi:10.1016/j.jpeds.2016.11.021

32. Gururaj G, Varghese M, Benegal V. National Mental Health Survey, Chhattisgarh State Report 2015-16:
Prevalence, Pattern and Outcomes. http://indianmhs. nimhans.ac.in/Docs/statereports/chhattisgarh-NMHSReport.pdf. Accessed July 22, 2019.

33. World Health Organization. Global Adult Tobacco Survey: Fact sheet, Chhattisgarh 2016-17. http://www.tiss.edu/ uploads/files/Chhattisgarh.pdf. Accessed July 22, 2019.

34. Indian Council of Medical Research. Tobacco related cancers. In: Consolidated Report of the HBCRs: 2012-2014. http://www.ncdirindia.org/ncrp/ALL_ NGRP_REPORTS/HBCR_REPORT_2012_2014/ ALL_CONTENT/PDF_Printed_Version/Chapter3.pdf. Accessed July 22, 2019.

35. Gupta B, Johnson NW. Systematic review and metaanalysis of association of smokeless tobacco and of betel quid without tobacco with incidence of oral cancer in South Asia and the Pacific. PLoS One. 2014;9(11):e113385. doi:10.1371/journal.pone.0113385

36. Miller WR, Rollnick S. Motivational Interviewing: Preparing People for change. New York, NY: The Guilford Press; 2002.

37. Reid RD, Mullen K-A, Slovinec D'Angelo ME, Aitken DA, Papadakis S, Haley PM, et al. Smoking cessation for hospitalized smokers: An evaluation of the 'Ottawa Model.' Nicotine Tob Res. 2010;12(1):11-18. doi:10.1093/ntr/ntp165

38. Papadakis S, Cole AG, Reid RD, Coja M, Aitken D, Mullen K-A, et al. Increasing Rates of Tobacco Treatment Delivery in Primary Care Practice: Evaluation of the Ottawa Model for Smoking Cessation. Ann Fam Med. 2016;14(3):235243. doi:10.1370/afm.1909

39. Heatherton TF, Kozlowski LT, Frecker RC, Fagerström KO. The Fagerström test for nicotine dependence: A revision of the Fagerström tolerance questionnaire. Br J Addict. 1991;86(9):1119-1127. doi:10.1111/j.1360-0443.1991.tb01879.x

40. Ebbert JO, Patten CA, Schroeder DR. The Fagerström Test for Nicotine Dependence-Smokeless Tobacco (FTND-ST). Addict Behav. 2006;31(9):1716-1721. doi:10.1016/j.addbeh.2005.12.015

41. Ahsan M, Saad M, Jawed M, Akhtar S, Haseeb A, Bilal M, Ahmed A. Impact of tobacco health warnings on smokers in Pakistan. J Pak Med Assoc. 2016; 66(1):5962. PMID:26712183.

42. Amin MB, Edge S, Greene F, Byrd DR, Brookland RK, Washington MK et al. AJCC Cancer Staging Manual. 8th ed. New York, NY: Springer; 2017.

43. Piccirillo JF, Tierney RM, Costas I, Grove L, Spitznagel, Jr EL. Prognostic Importance of Comorbidity in a HospitalBased Cancer Registry. JAMA. 2004;291(20):2441-2447. doi:10.1001/jama.291.20.2441

44. Deshpande NC, Nawathe AA. Translation and validation of Hindi version of Oral Health Impact Profile-14. J Indian Soc Periodontol. 2015;19(2):208-210. doi:10.4103/0972-124X.145806

45. Slade GD. Derivation and validation of a short-form oral 
health impact profile. Community Dent Oral Epidemiol. 1997;25(4):284-290. doi:10.1111/j.1600-0528.1997.tb00941

CONFLICTS OF INTEREST

The authors have each completed and submitted an ICMJE form for disclosure of potential conflicts of interest. The authors declare that they have no competing interests, financial or otherwise, related to the current work. All authors report that this research received funding from Chhattisgarh State Planning Commission.

\section{FUNDING}

This research received funding from Chhattisgarh State Planning Commission, 1387/SPC/SNo/2017-18 Dt 28/11/2018.

PROVENANCE AND PEER REVIEW

Not commissioned; externally peer reviewed. 